\title{
Establishing A Regional Management Pathway for Peri-Anal and Anal Cancers and Pre-Cancers in a Moderate Prevalence HIV Setting
}

\author{
D Ogbonmwan, J Hussey \\ Genito-Urinary Medicine Department, Sunderland Royal Hospital, \\ City Hospitals Sunderland NHS Foundation Trust
}

\section{Introduction}

There is a rising incidence of anal cancer in the UK. ${ }^{1}$ People living with HIV (PLWH) are at an increased risk of anal cancer and pre-cancer, and this is especially so amongst men who have sex with men (MSM). Sexual transmission of high risk Human Papilloma Virus (HPV), smoking and older age are contributing factors. Screening for anal cancer has not been found to be cost effective by some groups ${ }^{2}$ and a wide difference in screening or testing methods exists nationally. BHIVA guidelines state centres should incorporate a pathway of managing suspected peri-anal and anal cancers and precancers. $^{3}$

\section{Methods}

Our aim was to collate data on current screening and referral methods for peri-anal and anal lesions within our region to guide establishing a regional management pathway.

An online survey was sent to specialists involved in managing $\mathrm{PLWH}$. This included trainees and Consultants in Infectious Disease and Genito-urinary medicine (GUM). They were asked the methods used, if any, in routine clinics for identifying PLWH with anal and perianal cancers and pre-cancers, and whether there was a local established management pathway.

\section{$\underline{\text { Results }}$}

23 responses to the survey were received, largely from within GUM. 65\% of respondents were Consultants. 33\% of respondents stated that they regularly screened PLWH for peri-anal and anal lesions; the majority by enquiring about symptoms or carrying out proctoscopy examination, largely in men who have sex with men and PLWH with known anogenital warts. Only one Infectious Diseases specialist felt comfortable in using a proctoscope, and $67 \%$ of clinicians did not feel that they could be involved in the annual surveillance of peri-anal and anal intra-epithelial neoplasia.

\section{References}

1. Cancer Research UK http://www.cancerresearchuk.org/healthprofessional/cancer-statistics/statistics-by-cancer-type/analcancer/incidence\#heading-Two [Accessed May 2017]

2. Czoski-Murray C, Karnon J, Jones R, Smith K, Kinghorn G. Costeffectiveness of screening high risk HIV-positive men who have sex with men (MSM) and HIV-positive women for anal cancer. Health Technol Assess 2010;14(53).

3. Fakoya A, Lamba $\mathrm{H}$, Mackie $\mathrm{N}$ et al British HIV Association, BASHH and FSRH guidelines for the management of the sexual and reproductive healt of people living with HIV infevtion. HIV Medicine 2008;9(681-720)

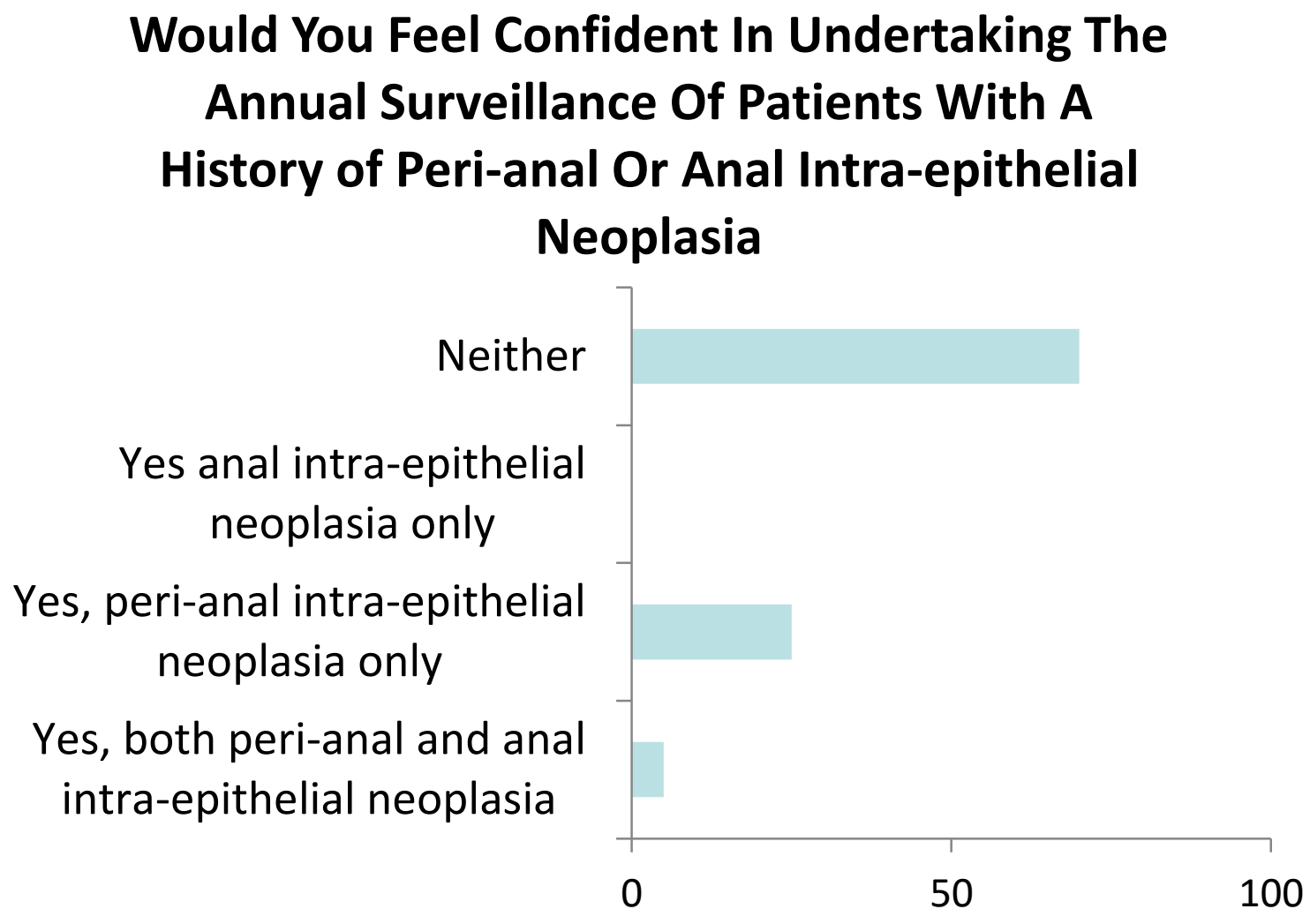

Do You Have A Local Referral Pathway For Patients With Suspicious Peri-anal Or Anal Lesions

No clear referral pathway
Yes, biopsy to be done by
colorectal surgeons
Yes, following in house
biopsy 0

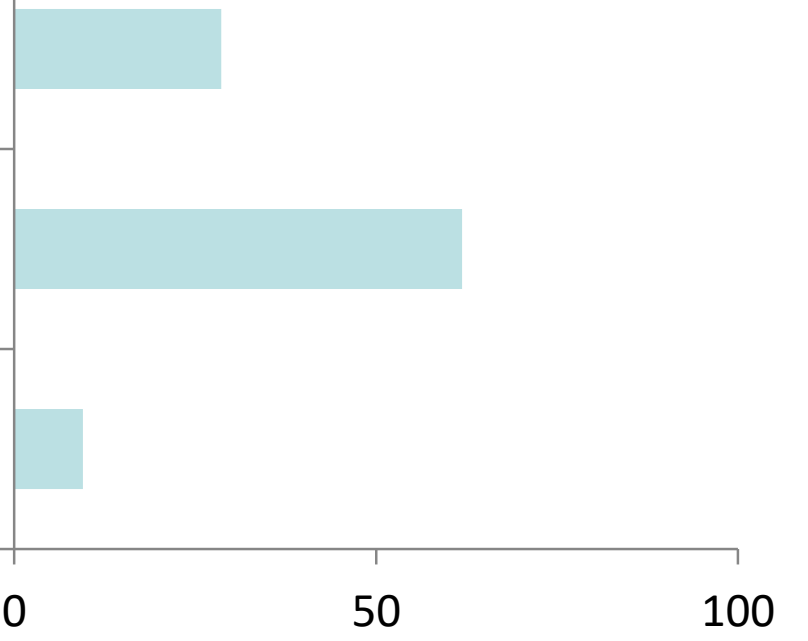

\section{Discussion}

The results have supported the need for the implementation of a peri-anal and anal cancer and precancer management pathway within our HIV regional network (see below), alongside further education and streamlining of screening within the region.

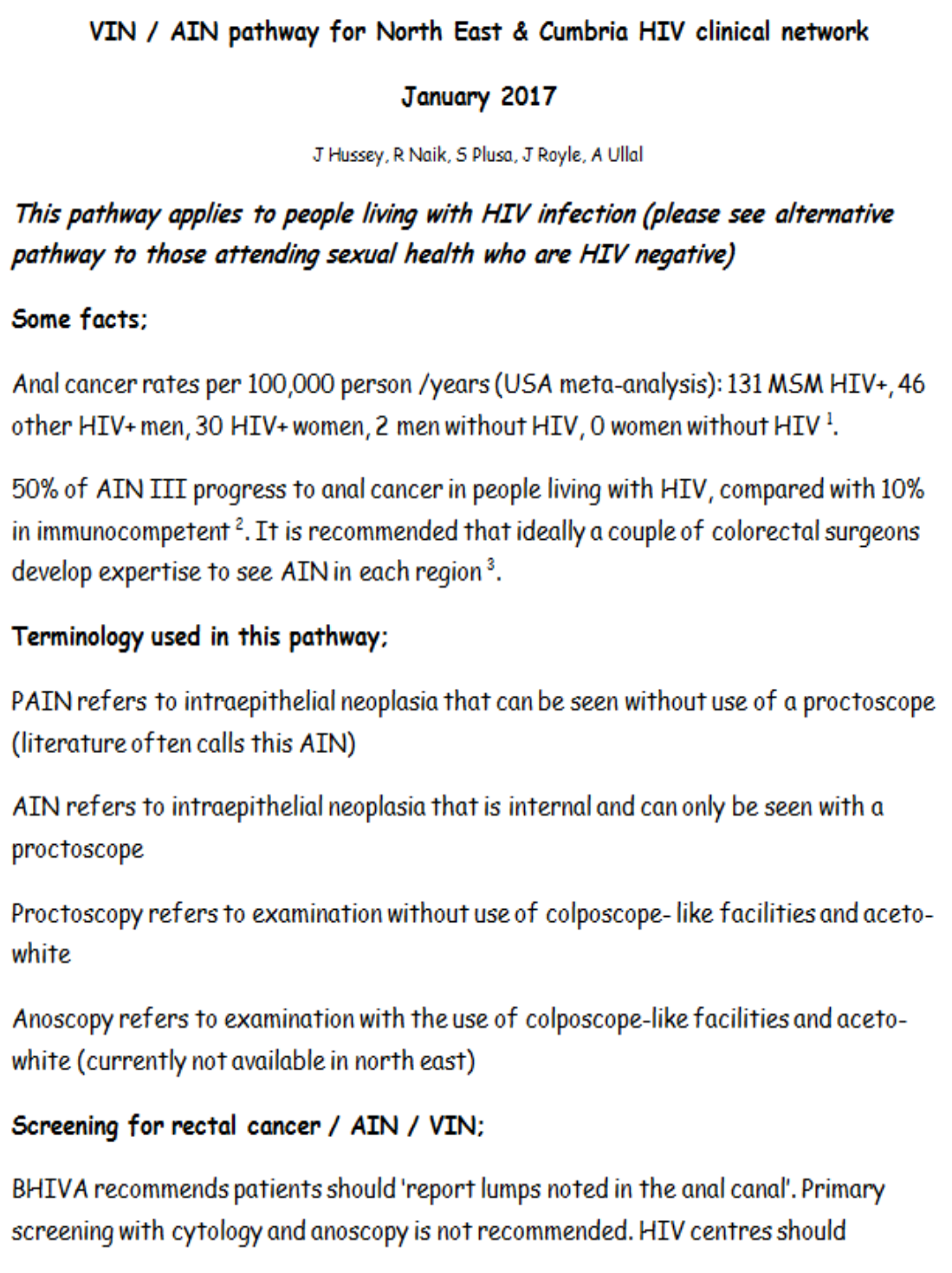

